\title{
Research Article \\ Fuzzy Type Relations and Transformation Operators Defined by Monads
}

\author{
Jiří Močkoř \\ University of Ostrava, Center of Excellence IT4Innovations 30. dubna 22, 70103 Ostrava 1, Czech Republic
}

\section{ARTICLE INFO}

Article History

Received 23 Mar 2020

Accepted 17 Sep 2020

Keywords

Powerset object

monad

Monadic relation

Transformation operator defined by monadic relation

\begin{abstract}
Using the theory of monads in categories and the theory of monadic relations, the concept of general transformation operator defined by a monadic relation is introduced. It is proven that a number of standard relations used in categories of fuzzy structures are monadic relations for monads defined in these categories. It is also proven that a number of standardly used transformation operators in fuzzy sets, fuzzy rough sets, or fuzzy soft sets are in the form of general operators defined by monadic relations.
\end{abstract}

(C) 2020 The Authors. Published by Atlantis Press B.V. This is an open access article distributed under the CC BY-NC 4.0 license (http://creativecommons.org/licenses/by-nc/4.0/).

\section{INTRODUCTION}

Fuzzy set theory was introduced by Zadeh [1] as a generalization of the classical set theory, allowing working with vagueness, as one of the basic features of real-world applications. Concurrently with its origins, the theory of fuzzy sets dealt not only with objects, i.e., with fuzzy sets, but also investigated the functional relations between these objects. This naturally led to research into the categorical aspects of fuzzy sets and, in general, to introduction of various categories of fuzzy sets structures. The fuzzy set categories were often designed to be as close as possible to the classical category of sets. This led to an effort to create such categories of fuzzy sets that would be topos [2-5]. With the development of the fuzzy set theory and applications, the Heyting algebra was gradually abolished as an array of fuzzy set values and replaced by other complete lattice structures, such as the totally monoidal sets and various generalizations of these structures (see [3,6-14] and others). However, it was still valid that the key role of morphisms in these new categories had two main structures, namely the mappings between underlying sets of fuzzy sets with specific properties on the one hand and the fuzzy relations (again with special properties) on the other.

Recently, however, a number of results have emerged in the theory of fuzzy sets, which are based on the application of fuzzy relations in suitable categories. A typical example of this use of fuzzy relations is a category $\mathbf{K}$ of sets as objects and $\mathcal{L}$-valued fuzzy relations between sets as morphisms, where $\mathcal{L}$ is a complete residuated lattice. This category is frequently used in various powerset functors $T: \mathbf{K} \rightarrow$ CSLAT, where CSLAT is the category of complete semilattices with

\footnotetext{
*Email: mockor@osu.cz
}

semilattices homomorphisms. These powerset functors then represent generalizations of Zadeh's extension principle, where instead of extensions of maps between two sets, general morphisms (including various fuzzy relations) are used. The relational variant of Zadeh's extension was for the first time defined by Goguen [15], when he introduced the notion of the image of a fuzzy set under a fuzzy relation. Many examples using explicitly or implicitly powerset functors can be found in rough fuzzy sets theory, F-transform theory and many others (see, e..g., [16-19]). Powerset functors that convert morphisms $f: X \rightarrow Y$ between two fuzzy objects from a category to mappings between some sets of corresponding fuzzy objects $T(X)$ and $T(Y)$ defined above these objects sometimes represent also special types of transformation operators. Typical examples of such transformation operators are of the form $\mathcal{L}^{X} \rightarrow \mathcal{C}(Y)$, where $\mathcal{C}(Y) \subseteq \mathcal{L}^{Y}$ is a set of fuzzy sets with some special properties. Therefore, fuzzy sets from $\mathcal{L}^{X}$ are approximated by special fuzzy sets from $\mathcal{C}(Y)$. A common feature of a large part of these transformation operators is that for individual fuzzy structures these transformation operators are defined individually and thus we have always to check their properties. This individual approach also prevents the possibility of creating relationships between individual operators or effectively transforming one type of operators to another.

In this paper, we will therefore focus on the issue of general transformation operators in categories, which, however, would include a large proportion of transformation operators normally used in various fuzzy structures. We show that there are a number of transformation operators that can be defined using the same formula in different categories with objects made up of fuzzy structures and morphisms defined by relations of different types. These transformation operators are based on the theory of relations defined 
by monads, using the idea of Manes [20]. In that case, a relation is not defined absolutely, it is tied to the existence of a monad in the category. Therefore to different monads $\mathbf{T}$ in a category $\mathbf{K}$, different types of relations between objects in this category, called $\mathbf{T}$-relations, can be defined in $\mathbf{K}$. We prove that large part of relations which are standardly used in fuzzy sets theory are, in fact, monadic relations in suitable categories.

Using these results, we introduce the notion of transformation operators defined by monadic relations. These transformation operators transform a structure $T(X)$ defined over an object $X$ of a category $\mathbf{K}$ to the structure $T(Y)$ defined over an object $Y$, where $\mathbf{T}$ is a monad in a category $\mathbf{K}$ and where the transformation process is defined by a monadic T-relation from $X$ to $Y$.

We show that this generally defined transformation operator include a number of known and often used transformations in fuzzy sets and fuzzy rough sets theory, including various generalizations of Zadeh's extension principle, transformations defined using lattice-valued fuzzy relations, and lattice-valued fuzzy transform. Although the definitions of these operators are different and relate to different fuzzy structures, we show that all these transformation operators are special examples of generally defined transformation operators for various monadic relations.

This universal approach to the construction of transformation operators of this type makes it possible to unify the theory of these operators, allows by the selection of appropriate categories and monads in these categories to create new transformation operators, and using morphisms between monads it allows to find relationships between individual transformation operators.

A typical example of this type of transformation is the F-transform operator, which converts a certain fuzzy relation in $R: X \times I \rightarrow \mathcal{L}$ to the map $F(R): \mathcal{L}^{X} \rightarrow \mathcal{L}^{I}$, where $X$ is a set and $I$ is an index set of a fuzzy partition of the set $X$. That is, $F(R)$ approximates fuzzy sets from $\mathcal{L}^{X}$ into fuzzy sets defined above smaller index set $I$.

All these results make it possible to understand monads in fuzzy object categories as another useful tool in fuzzy set theory. This tool not only makes it possible to create new examples of the abovementioned terms but also creates a common basis for many previously isolated constructions in fuzzy set theory.

\section{PRELIMINARIES}

In this section we introduce principal notions and categories based on fuzzy sets which we use in the paper. A principal lattice structure used in fuzzy set theory in the paper is a complete residuated lattice (see e.g., [21]), i.e., a structure $\mathcal{L}=\left(L, \wedge, \vee, \otimes, \rightarrow, 0_{L}, 1_{L}\right)$ such that $(L, \wedge, \vee)$ is a complete lattice $\left(L, \otimes, 1_{L}\right)$, is a commutative monoid with operation $\otimes$ isotone in both arguments and $\rightarrow$ is a binary operation which is residuated with respect to $\otimes$, i.e.,

$$
\alpha \otimes \beta \leqslant \gamma \quad \text { iff } \quad \alpha \leqslant \beta \rightarrow \gamma .
$$

If $\mathcal{L}$ is a complete residuated lattice, a $\mathcal{L}$-fuzzy set in a crisp set $X$ is a map $f: X \rightarrow L$. $f$ is a nontrivial $\mathcal{L}$-fuzzy set, if $f$ is not identical to the zero function. The core of a $\mathcal{L}$-fuzzy set $f$ in a set $X$ is defined by $\operatorname{core}(f)=\left\{x \in X: f(x)=1_{L}\right\}$.
A set with $\mathcal{L}$-valued similarity relation (or $\mathcal{L}$-set) is a couple $(A, \delta)$, where $A$ is a set and $\delta: A \times A \rightarrow L$ is a map such that
(a) $(\forall x \in A) \quad \delta(x, x)=1$,
(b) $(\forall x, y \in A) \quad \delta(x, y)=\delta(y, x)$,
(c) $(\forall x, y, z \in A) \delta(x, y) \otimes \delta(y, z) \leqslant \delta(x, z)$ (generalized transitivity).

In the paper we use some standard categories with (sometimes special) maps as morphisms. Namely,

1. Category CSLAT of complete $\vee$-semilattices as objects and with semilattice homomorphisms as morphisms.

2. Category Set of sets as objects with mappings as morphisms.

3. Category $\operatorname{Set}(\mathcal{L})$ with $\mathcal{L}$-sets $(X, \delta)$ as objects and with $f:(X, \delta) \rightarrow(Y, \gamma)$ as morphisms, where $f: X \rightarrow Y$ is a map and $\delta(x, y) \leqslant \gamma(f(x), f(y)), x, y \in X$.

4. Category SpaceFP of spaces with fuzzy partitions and corresponding morphisms.

Within morphisms in the category $\operatorname{Set}(\mathcal{L})$, we will be specially interested in morphisms $(A, \delta) \rightarrow(L, \leftrightarrow)$, where $\leftrightarrow$ is the biresiduation operator in $\mathcal{L}$. These morphisms are called fuzzy sets in $A$ extensional with respect to $\delta$, Hence, $f:(A, \delta) \rightarrow L$ is an extensional fuzzy sets if $f(x) \otimes \delta(x, y) \leqslant f(y)$, for arbitrary $x, y \in A$. By $F(A, \delta)$ we denote the set of all fuzzy sets in A extensional with respect to $\delta$.

Besides these well-known categories we use also the category SpaceFP of spaces with fuzzy partitions which was introduced in [22] and which is the basic category for lattice-valued F-transforms. In the next part we recall the definition of the category SpaceFP, which is based on $\mathcal{L}$-valued fuzzy partitions, as it was introduced in [23] and some additional facts about objects and morphisms of that category.

Definition 1. [23] Let $X$ be a set. A system $\mathcal{A}=\left\{A_{\lambda}: \lambda \in \Lambda\right\}$ of normal $\mathcal{L}$-valued fuzzy sets in $X$ is a fuzzy partition of $X$, if $\left\{\operatorname{core}\left(A_{\lambda}\right): \lambda \in \Lambda\right\}$ is a partition of $X$. A pair $(X, \mathcal{A})$ is called a space with a fuzzy partition. The index set of $\mathcal{A}$ will be denoted by $|\mathcal{A}|$.

Definition 2. [22] The category SpaceFP is defined by

1. Fuzzy partitions $(X, \mathcal{A})$, as objects,

2. Morphisms $(g, \sigma):\left(X,\left\{A_{\lambda}: \lambda \in \Lambda\right\}\right) \rightarrow\left(Y,\left\{B_{\omega}: \omega \in \Omega\right\}\right)$, such that
(a) $g: X \rightarrow Y$ and $\sigma: \Lambda \rightarrow \Omega$ are maps,
(b) $\forall \lambda \in \Lambda, A_{\lambda}(x) \leqslant B_{\sigma(\lambda)}(g(x))$, for each $x \in X$.

3. The composition of morphisms in SpaceFP is defined by $(h, \tau) \circ(g, \sigma)=(h \circ g, \tau \circ \sigma)$.

In the lattice $\mathcal{L}$ we can define a special fuzzy partition $\mathcal{Q}=\left\{Q_{\alpha}: \alpha \in\right.$ $L\}$, where $Q_{\alpha}(\beta)=\alpha \leftrightarrow \beta$ and $\leftrightarrow$ is the bi-residuum operation in $\mathcal{L}$. Then $(\mathcal{L}, \mathcal{Q})$ is a space with a fuzzy partition. The following notion of an extensional fuzzy set in the category SpaceFP extends the notion of an extensional fuzzy set in the category $\operatorname{Set}(\mathcal{L})$. 
Definition 3. A map $f: X \rightarrow L$ is an extensional fuzzy set in a space with a fuzzy partition $(X, \mathcal{A})$ in the category SpaceFP, if there exists a map $\sigma:|\mathcal{A}| \rightarrow L$, such that $(f, \sigma)$ is a morphism $(X, \mathcal{A}) \rightarrow(\mathcal{L}, \mathcal{Q})$ in the category SpaceFP. By $R(X, \mathcal{A})$ we denote the set of all extensional fuzzy sets in $(X, \mathcal{A})$.

In the paper [24] we proved that for arbitrary space with a fuzzy partition $(X, \mathcal{A})$ there exists $\mathcal{L}$-valued similarity relation $\delta_{X, \mathcal{A}}$ (called a characteristic similarity relation of $(X, \mathcal{A})$ ) defined on $X$ such that $f: X \rightarrow L$ is extensional in $(X, \mathcal{A})$ if and only if $f:\left(X, \delta_{X, \mathcal{A}}\right) \rightarrow L$ is an extensional fuzzy set. Therefore,

$$
R(X, \mathcal{A})=F\left(X, \delta_{X, \mathcal{A}}\right)
$$

\section{RELATIONS DEFINED BY MONADS}

For a variety of classic categories where morphisms are various types of mappings, including categories $\operatorname{Set}, \operatorname{Set}(\mathcal{L})$ and $\operatorname{SpaceFP}$, there are a number of variants where various types of relations and fuzzy relations can be used as morphisms instead of mappings. Let us consider the following examples of these relations in well-known categories.

Example 1. A binary relation $R \subseteq X \times Y$ from $X$ to $Y$ in the category Set with the standard composition $S \circ R$ of binary relations.

Example 2. An $\mathcal{L}$-valued fuzzy relation $R: X \times Y \rightarrow L$ from a set $X$ to a set $Y$ in the category Set with a composition or relations $R: X \rightarrow Y$ and $S: Y \rightarrow Z$ is defined by

$$
\forall x \in X, z \in Z, \quad S \circ R(x, z)=\bigvee_{y \in Y} R(x, y) \otimes S(y, z)
$$

Example 3. A relation $R$ from an $\mathcal{L}$-set $(X, \delta)$ to the $\mathcal{L}$-set $(Y, \gamma)$ in the category $\operatorname{Set}(\mathcal{L})$ is an $\mathcal{L}$-valued fuzzy relation $R: X \times Y \rightarrow L$ such that

$$
\begin{aligned}
& R(x, y) \otimes \delta\left(x, x^{\prime}\right) \leqslant R\left(x^{\prime}, y\right), \\
& R(x, y) \otimes \gamma\left(y, y^{\prime}\right) \leqslant R\left(x, y^{\prime}\right) .
\end{aligned}
$$

and with a composition $S \circ R$ of relations defined the in the same way as in the Example 2.

Example 4. A relation from a space with a fuzzy partition $(X, \mathcal{A})$, $\mathcal{A}=\left\{A_{i}: i \in|\mathcal{A}|\right\}$, to another space with a fuzzy partition $(Y, \mathcal{B})$, $\mathcal{B}=\left\{B_{j}: j \in|\mathcal{B}|\right\}$ in the category SpaceFP is a fuzzy relation $R: X \times Y \rightarrow L$ such that $R$ is a relation from the $\mathcal{L}$-set $\left(X, \delta_{X, \mathcal{A}}\right)$ to the $\mathcal{L}$-set $\left(Y, \delta_{Y, \mathcal{B}}\right)$ according to the Example 3, where $\delta_{X, \mathcal{A}}$ and $\delta_{Y, \mathcal{B}}$ are characteristic similarity relations of spaces with fuzzy partitions. It follows that $R$ satisfies the following inequalities for arbitrary $x, x^{\prime} \in X, y, y^{\prime} \in Y$ :

$$
\begin{aligned}
& R(x, y) \otimes \delta_{X, \mathcal{A}}\left(x, x^{\prime}\right) \leqslant R\left(x^{\prime}, y\right), \\
& R(x, y) \otimes \delta_{Y, \mathcal{B}}\left(y, y^{\prime}\right) \leqslant R\left(x, y^{\prime}\right) .
\end{aligned}
$$

Example 5. We use the following notion of a fuzzy soft set, introduced in [25]:

Let $X$ and $U$ be sets. A pair $(E, s)$ is called a L-valued fuzzy soft set in a space $(X, U)$, if $E \subseteq X$ and $s: E \rightarrow L^{U}$.

A fuzzy set $s(e) \in L^{U}$, where $e \in E$, represents "fuzzy" evaluation of how individual objects in $U$ correspond to the parameter $e$ of the set of parameters $E$, i.e., $s(x)(u) \in L$ is the $L$-valued measure of how the object $u \in U$ corresponds to parameter $e \in E$. In what follows, we assume that the set $U$ is constant (i.e., fixed). So we will only deal with fuzzy soft sets that evaluate a fixed set $U$ of objects with different sets of criteria. In that case, instead of a space $(X, U)$ we will talk about a set $X$ only.

A fuzzy soft relation from a set $X$ to $Y$ was defined in [26] as a fuzzy soft set $(E, R)$ in a set $X \times Y$, i.e., $E \subseteq X \times Y$ and $R: E \rightarrow L^{U}$. The composition $\oplus$ of fuzzy soft relations $(E, R)$ from $X$ to $Y$ and $(F, S)$ from $Y$ to $Z$ is defined by

$$
\begin{gathered}
(F, S) \oplus(E, R)=(F . E, S \circ R), \\
F . E=\left\{(x, z) \in X^{2}: \exists y \in X,(x, y) \in E,(y, z) \in F\right\}, \\
(x, z) \in F . E,(S \circ R)(x, z)(u)=\bigvee_{\{y \mid(x, y) \in E,(y, z) \in F\}} R(x, y)(u) \otimes S(y, z)(u),
\end{gathered}
$$

for arbitrary $u \in U$.

Whatever the Examples 1-5 of relations between objects in some categories seem to be rather natural, it is possible to ask why these definitions of relations are chosen and not the other ones. In order to answer this question, we should set out clear rules on what can be such a definition of a relation or a fuzzy relation in a category.

As we mentioned in the Introduction, one of the possibilities how to define relations in a category is to use the approach introduced by Manes in his published review [20]. In this concept of relations in a category $\mathbf{K}$, a relation is not defined absolutely, it is tied to the existence of a monad in this category. Therefore to different monads $\mathbf{T}$ in a category $\mathbf{K}$, different types of relations between objects in this category, called $\mathbf{T}$-relations, can be defined in $\mathbf{K}$. This approach allows to link the theory of categories with relations as morphisms with more general structures, i.e., monads in categories, while at the same time allowing to justify the more general nature of the choice of the specific type of relations in the category representing morphisms.

For the convenience of readers we firstly repeat basic definitions of monads and corresponding Kleisli categories, which are necessary for introduction of monadic relations in a category. The idea of using monads for fuzzification is based on extension of objects $X$ of a category $\mathbf{K}$ to another object $T(X) \in \mathbf{K}$, which may be regarded as a "cloud of fuzzy states" with a morphism $\eta: X \rightarrow T(X)$, representing "crisp" states in the object of "fuzzy states." Then a "fuzzy morphism" $f: X \rightsquigarrow Y$ is simply a morphism $f: X \rightarrow T(Y)$ in the category $\mathbf{K}$ and a composition of fuzzy morphisms is defined by a special operation $\diamond$. The result of these constructions is a triple $\mathbf{T}=(T, \diamond, \eta)$, which Manes [27] called a fuzzy theory and which, in fact, is a monad (or algebraic theory) in a clone form.

Definition 4. $\mathbf{T}=(T, \diamond, \eta)$ is a $\operatorname{monad}$ (in clone form) in a category $\mathbf{K}$, if

1. $T: \mathbf{K} \rightarrow \mathbf{K}$ is an object function.

2. $\eta$ is a system of $\mathbf{K}$-morphisms $\eta_{A}: A \rightarrow T(A)$, for any object $A$.

3. For each pair of K-morphisms $f: A \rightarrow T(B), g: B \rightarrow T(C)$, there exists a composition (called a Kleisli composition) $g \diamond f: A \rightarrow$ $T(C)$, which is associative.

4. For every K-morphism $f: A \rightarrow T(B), \eta_{B} \diamond f=f$. 
5. $\diamond$ is compatible with composition of morphisms of $\mathbf{K}$, i.e., for all K-morphisms $f: A \rightarrow B, g: B \rightarrow T(C)$, we have $g \diamond\left(\eta_{B} . f\right)=$ $g . f$.

For a monad $\mathbf{T}$ in a category $\mathbf{K}$ a new category $\mathbf{K}_{\mathbf{T}}$ can be defined, called the Kleisli category. We recall a definition of $\mathbf{K}_{\mathbf{T}}$ (see, e.g., $[27,28])$.

Definition 5. Let $\mathbf{K}$ be a category and let $\mathbf{T}=(T, \diamond, \eta)$ be a monad (in clone form) in $\mathbf{K}$. Then the Kleisli category $\mathbf{K}_{\mathbf{T}}$ of $\mathbf{T}$ is defined by

1. Objects of $\mathbf{K}_{\mathbf{T}}=$ objects of $\mathbf{K}$.

2. For any objects $A, B \in \mathbf{K}, \operatorname{Hom}_{\mathbf{K}_{\mathbf{T}}}(A, B)=\operatorname{Hom}_{\mathbf{K}}(A, T(B))$. Morphisms in $\mathbf{K}_{\mathbf{T}}$ are denoted by $A \rightsquigarrow B$.

3. A composition of morphisms $f: A \rightsquigarrow B, g: B \rightsquigarrow C$ is defined by $g \diamond f$.

According to Manes, we can define the notion of a monadic T-relation in a category.

Definition 6. Let $\mathbf{K}$ be a category and let $\mathbf{T}=(T, \diamond, \eta)$ be a monad in $\mathbf{K}$.

1. A monadic $\mathbf{T}$-relation $R$ from an object $X$ to an object $Y$ in $\mathbf{K}$ is a morphism $R: X \rightsquigarrow Y$ in the Kleisli category $\mathbf{K}_{\mathbf{T}}$, or, equivalently, the morphism $R: X \rightarrow T(Y)$ in the category $\mathbf{K}$.

2. If $R: X \rightsquigarrow Y$ and $S: Y \rightsquigarrow Z$ are monadic T-relations, their composition is a T-relation $S \diamond R: X \rightsquigarrow Z$.

From the above definition it follows that the Kleisli category $\mathbf{K}_{\mathbf{T}}$ can be considered the relational variant of a category $\mathbf{K}$ where instead of morphisms of category K, T-relations are used.

To justify the examples of relations presented in Examples 1-5 we show that all these relations are, in fact, monadic $\mathbf{T}$-relations for appropriate monads in the corresponding categories. It follows that the relational versions of categories from these examples, where instead of classical maps as morphisms, relations from these examples are considered, are isomorphic to the Kleisli categories of original categories with respect to appropriate monads.

Theorem 1. Let us consider an arbitrary example from Examples 1-5. Then there exists a monad $\mathbf{T}=(T, \diamond, \eta)$ in the category $K$ of this example such that

1. There is a bijection $\Phi$ between the set of relations defined by this example and the set of monadic $T$-relations in this category $K$.

2. If $S \cdot R$ is a composition of relations from this Example, then we have

$$
\Phi(S \cdot R)=\Phi(S) \diamond \Phi(R)
$$

Proof. Monads $\mathbf{T}$ in corresponding categories $\mathbf{K}$ for Examples 1-5 were introduced in various papers. Recall [14,27,29] for monads $\mathbf{P}$ and $\mathbf{Z}$ in the category Set [30], for the monad $\mathbf{F}$ in the category $\operatorname{Set}(\mathcal{L})$ [31], for the monad $\mathbf{R}$ in the category SpaceFP and [26] for the monad $\mathbf{T}$ in the category Set. We repeat definitions of these monads for corresponding examples and we prove the existence of the bijection $\Phi$ with corresponding properties.

Case Example 1: The monad $\mathbf{P}=(P, \diamond, \eta)$ in the category Set is defined by

1. For each object $X \in \operatorname{Set}, P(X)=2^{X}$,

2. For each object $X \in$ Set, $\eta_{X}: X \rightarrow P(X)$ by $\eta_{X}(x)=\{x\}$.

3. For each $f: X \rightarrow P(Y), g: Y \rightarrow P(Z), g \diamond f: X \rightarrow P(Z)$, by

$$
(g \diamond f)(x)=\bigcup_{y \in f(x)} g(y) .
$$

If $R$ is a relation from $X$ to $Y$, then $\Phi(R): X \rightarrow P(Y)$ is defined by $\Phi(R)(x)=\{y \in Y:(x, y) \in R\}$. It is clear that $\Phi$ is a bijection and $\Phi(S \circ R)=\Phi(s) \diamond \Phi(R)$.

Case Example 2: The monad $\mathbf{Z}=(Z, \diamond, \chi)$ in the category Set is defined by

1. For each object $X \in$ Set, the object function $Z$ : Set $\rightarrow$ Set is defined by $Z(X)=L^{X}$,

2. For each object $X \in$ Set, $\chi_{X}: X \rightarrow L^{X}$ is a characteristic map $\chi_{X,\{x\}}: X \rightarrow L$ of $\{x\}$ in $X$,

3. For each $f: X \rightarrow L^{Y}$ and $g: Y \rightarrow L^{Z}$ in Set, $g \diamond f: X \rightarrow L^{Z}$ is defined by

$$
x \in X, z \in Z, \quad(g \diamond f)(x)(z)=
$$

$$
\bigvee_{y \in Y} f(x)(y) \otimes g(y)(z)
$$

If $R$ is a relation from $X$ to $Y$, then $\Phi(R): X \rightarrow Z(Y)$ is defined by $\Phi(R)(x)(y)=R(x, y)$. It is clear that $\Phi$ is a bijection and $\Phi(S \circ R)=$ $\Phi(S) \diamond \Phi(R)$.

Case Example 3: The monad $\mathbf{F}=(\mathcal{F}, \diamond, \eta)$ in the category $\operatorname{Set}(\mathcal{L})$ is defined by

1. The object function $\mathcal{F}: \operatorname{Set}(\mathcal{L}) \rightarrow \operatorname{Set}(\mathcal{L})$ is $\operatorname{defined}$ by $\mathcal{F}(X, \delta)=\left(F(X, \delta), \sigma_{X, \delta}\right)$, where the similarity relation $\sigma$ is defined by

$$
\sigma_{X, \delta}(s, t)=\bigwedge_{x \in X} s(x) \leftrightarrow t(x),
$$

for all $s, t \in F(X, \delta)$.

2. For each $(X, \delta) \in \operatorname{Set}(\mathcal{L}), \eta_{(X, \delta)}:(X, \delta) \rightarrow \mathcal{F}(X, \delta)$ is defined by $\eta_{(X, \delta)}(a)(x)=\delta(a, x)$, for all $a, x \in X$.

3. For each $f:(X, \delta) \rightarrow \mathcal{F}(Y, \gamma)$, for each $g:(Y, \gamma) \rightarrow \mathcal{F}(Z, \omega)$ in $\operatorname{Set}(\mathcal{L}), g \diamond f:(X, \delta) \rightarrow \mathcal{F}(Z, \omega)$ is defined in the same way as in (2).

Let the mapping $\Phi$ be defined formally as in the previous case. Hence, for a relation $R$ from $(X, \delta)$ to $(Y, \gamma)$ defined in Example 3, 
$\Phi(R):(X, \delta) \rightarrow\left(F(Y, \gamma), \sigma_{Y, \gamma}\right)$ is defined by $\Phi(R)(x)(y)=R(x, y)$. It is clear that $\Phi(R)(x) \in F(Y, \gamma)$ and we have

$$
\begin{gathered}
\sigma_{Y, \gamma}\left(\Phi(R)(x), \Phi(R)\left(x^{\prime}\right)\right)=\bigwedge_{y \in Y} \Phi(R)(x)(y) \leftrightarrow \Phi(R)\left(x^{\prime}\right)(y)= \\
\bigwedge_{y \in Y} R(x, y) \leftrightarrow R\left(x^{\prime}, y\right) \leqslant \delta\left(x, x^{\prime}\right) .
\end{gathered}
$$

Hence, $\Phi(R)$ is a morphism in $\operatorname{Set}(\mathcal{L})$ and $\Phi(R)$ is a monadic $\mathcal{F}$-relation. It can be proven easily that $\Phi$ is a bijection.

Case Example 4: The monad $\mathbf{R}=(\mathcal{R}, \diamond, \nu)$ in SpaceFP was constructed in [31] and it is defined by

1. Object function $\mathcal{R}:$ SpaceFP $\rightarrow$ SpaceFP, defined by

$$
\mathcal{R}(X, \mathcal{A})=\left(R(X, \mathcal{A}), \mathcal{C}_{X, \mathcal{A}}\right),
$$

where $\mathcal{C}_{X, \mathcal{A}}=\left\{C_{f}: f \in R(X, \mathcal{A})\right\}$ and $C_{f}(g)=\wedge_{x \in X} f(x) \leftrightarrow g(x)$.

2. For each object $(X, \mathcal{A}) \in \operatorname{SpaceFP}, \nu_{X, \mathcal{A}}=(e, \varepsilon):(X, \mathcal{A}) \rightarrow$ $\mathcal{R}(X, \mathcal{A})$ is a morphism in SpaceFP, such that

$$
e: X \rightarrow R(X, \mathcal{A}), \quad \varepsilon:|\mathcal{A}| \rightarrow\left|\mathcal{C}_{X, \mathcal{A}}\right|=R(X, \mathcal{A}),
$$

defined by

$$
\forall x, z \in X, \quad e(x)(z)=\delta_{X, \mathcal{A}}(x, z),
$$$$
\forall \lambda \in|\mathcal{A}|, z \in X, \quad \varepsilon(\lambda)(z)=\delta_{X, \mathcal{A}}(x, z),
$$

where $x \in \operatorname{core}\left(A_{\lambda}\right)$.

3. For each morphisms $(f, u):(X, \mathcal{A}) \rightarrow \mathcal{R}(Y, \mathcal{B})$ and $(g, v):$ $(Y, \mathcal{B}) \rightarrow \mathcal{R}(Z, D)$ in SpaceFP, the composition $(g, v) \diamond(f, u):$ $(X, A) \rightarrow \mathcal{R}(Z, D)$ is defined by $(g, v) \diamond(f, u)=(g \Delta f, v \nabla u)$, where

$$
\begin{gathered}
\forall x \in X, z \in Z, \quad(g \Delta f)(x)(z)=\bigvee_{y \in Y} f(x)(y) \otimes g(y)(z), \\
\forall \lambda \in \Lambda, z \in Z, \quad(v \nabla u)(\lambda)(z)=\bigvee_{y \in Y} u(\lambda)(y) \otimes v\left(\omega_{y}\right)(z),
\end{gathered}
$$

where $\omega_{y}$ is the unique index in $\mathcal{B}$, such that $y \in \operatorname{core}\left(B_{\omega_{y}}\right)$.

According to [31], $\mathbf{R}$ is a monad in the category SpaceFP. Let $R$ be a relation from $(X, \mathcal{A})$ to $(Y, \mathcal{B})$, defined in Example 4 . The morphism $\Phi(R)=(\bar{R}, \underline{R})$

$$
(\bar{R}, \underline{R}):(X, \mathcal{A}) \rightarrow \mathcal{R}(Y, \mathcal{B})=\left(R(Y, \mathcal{B}), \mathcal{C}_{Y, \mathcal{B}}\right)
$$

in SpaceFP is defined by

$$
\begin{gathered}
\bar{R}: X \rightarrow R(Y, \mathcal{B}), \quad \bar{R}(x)(y)=R(x, y), \\
\underline{R}:|\mathcal{A}| \rightarrow\left|\mathcal{C}_{Y, \mathcal{B}}\right|=R(Y, \mathcal{B}), \quad \underline{R}(i)(y)=R(z, y),
\end{gathered}
$$

where $z \in \operatorname{core}\left(A_{i}\right)$. Using properties of relations from Example 4 it follows that $\bar{R}(x)$ and $\underline{R}(i)$ are elements of $R(Y, \mathcal{B})$, for arbitrary $x \in X, i \in|\mathcal{A}|$. To prove that $(\bar{R}, \underline{R})$ is a morphisms we need to show that

$$
C_{\underline{R}(i)}(\bar{R}(x)) \geqslant A_{i}(x),
$$

for arbitrary $x \in X, i \in|\mathcal{A}|$, which follows directly from the properties of the relation $\delta_{X, \mathcal{A}}$ (see [24]). Similarly it can be proven that for arbitrary R-relation $(R, S):(X, \mathcal{A}) \rightarrow\left(R(Y, \mathcal{B}), \mathcal{C}_{Y, \mathcal{B}}\right), \Phi^{-1}(R, S)=$ $\bar{R}: X \times Y \rightarrow L, \bar{R}(x, y)=R(x)(y)$, is a relation from Example 4 .

For further details including the property $\Phi\left(R^{\prime} . R\right)=\Phi\left(R^{\prime}\right) \diamond \Phi(R)$ see the proof of [32]; Theorem 3.1.

Case Example 5: The monad $\mathbf{T}=(T, \diamond, \xi)$ in the category Set was constructed in [26] and it is defined by

1. Object function $T:$ Set $\rightarrow$ Set, defined by

$$
T(X)=\left\{(E, s): E \subseteq X, s: E \rightarrow L^{U}\right\}
$$

2. For each object $X \in$ Set, $\xi_{X}: X \rightarrow T(X)$ is a map such that

$$
\begin{array}{cl}
x \in X, & \xi_{X}(x)=\left(\{x\}, \eta_{x}\right), \quad \eta_{x}:\{x\} \rightarrow L^{U}, \\
& u \in U, \quad \eta_{x}(x)(u)=1_{L} .
\end{array}
$$

3. To show the construction of $\diamond$ we use the following notation. If $f: X \rightarrow T(Y)$ is a map, then for $x \in X$ we set $f(x)=\left(Y_{x}^{f}, f_{x}\right)$, where $Y_{x}^{f} \subseteq Y$ and $f_{x}: Y_{x}^{f} \rightarrow L^{U}$. Let $f: X \rightarrow T(Y)$ and $g: Y \rightarrow$ $T(Z)$ be maps. Then $g \diamond f: X \rightarrow T(Z)$ is defined by

$$
\begin{gathered}
x \in X, \quad(g \diamond f)(x)=\left(Z_{x}^{g \diamond f},(g \diamond f)_{x}\right), Z_{x}^{g \diamond f}=\bigcup_{y \in Y_{x}^{f}} Z_{y}^{g}, \\
(g \diamond f)_{x}: Z_{x}^{g \diamond f} \rightarrow L^{U}, a \in Z_{x}^{g \diamond f}, u \in U, \\
(g \diamond f)_{x}(a)(u)=\bigvee_{\left\{y \in Y_{x}^{f}: a \in Z_{y}^{g}\right\}} f_{x}(y)(u) \otimes g_{y}(a)(u) .
\end{gathered}
$$

In Močkoř and Hurtik [26] it was proven that $\mathbf{T}$ is a monad in Set. Let $(E, R)$ be a fuzzy soft relation from $X$ to $Y$ defined in Example 5 . The T-relation $\Phi(E, R): X \rightarrow T(Y)$ is defined by

$$
\begin{gathered}
x \in X, \quad \Phi(E, R)(x)=\left(Y_{x}^{E}, R_{x}\right) \in T(Y), \\
Y_{x}^{E}=\{y \in Y:(x, y) \in E\}, \\
\forall y \in Y_{x}^{E}, R_{x}(y)(u)=R(x, y)(u) .
\end{gathered}
$$

The definition of $R_{x}$ is correct, because $E=\bigcup_{x \in X}\{x\} \times Y_{x}^{E}$.

Conversely, let $S: X \rightarrow T(Y)$ be a T-relation from $X$ to $Y$. According to our notation, for $x \in X$, we have $S(x)=\left(Y_{x}^{S}, S_{x}\right) \in T(Y)$, where $Y_{x}^{S} \subseteq Y, S_{x}: Y_{x}^{S} \rightarrow L^{U}$. Then we put

$$
\begin{gathered}
\Phi^{-1}(S)=(F, Q), \text { where } \\
F=\bigcup_{x \in X}\{x\} \times Y_{x}^{S}, \quad Q: F \rightarrow L^{U}, \\
x \in X, y \in Y_{x}^{S}, u \in U, \quad Q(x, y)(u)=S_{x}(y)(u) .
\end{gathered}
$$

It can be proven easily that $\Phi$ and $\Phi^{-1}$ are inverse maps.

Let $(E, Q),(F, S)$ be fuzzy soft relations from $X$ to $Y$ and from $Y$ to $Z$, respectively. According to the definition of $\Phi$, we obtain

$\Phi(E, Q)=R: X \rightarrow T(Y), \quad \Phi(F, S)=N: Y \rightarrow T(Z)$, $\forall x \in X, y \in Y, \quad R(x)=\left(Y_{x}^{E}, R_{x}\right), \quad N(y)=\left(Z_{y}^{F}, N_{y}\right)$, $R_{x}(y)(u)=Q(x, y)(u), \quad N_{y}(Z)(u)=S(y, z)(u)$. 
Then, we obtain

$$
\begin{gathered}
x \in X, \quad(N \diamond R)(x)=\left(Z_{x}^{N \diamond R},(N \diamond R)_{x}\right), Z_{x}^{N \diamond R}=\bigcup_{y \in Y_{x}^{E}} Z_{y}^{F}, \\
z \in X_{x}^{N \diamond R}, \quad(N \diamond R)_{x}(z)=\bigvee_{\left\{y \in Y_{x}^{E} \mid z \in Z_{y}^{F}\right\}} R_{x}(y)(u) \otimes N_{y}(z)(u)= \\
\bigvee_{\{y \mid(x, y) \in E,(y, z) \in F\}} Q(x, y)(u) \otimes S(y, z)(u)=S \circ Q(x, z) .
\end{gathered}
$$

It follows that $\bigcup_{x \in X}\{x\} \times X_{x}^{s \diamond r}=F . E$ and

$$
\Phi(F, S) \diamond \Phi(E, Q)=N \diamond R=\Phi(F . E, S \circ Q)=\Phi((F, S) \oplus(E, Q)) .
$$

\section{TRANSFORMATION OPERATORS DEFINED BY MONADIC RELATIONS}

In classical mathematics and fuzzy mathematics, various kinds of transforms are used as powerful methods for construction of transformation models. The main idea of them consists in transforming an original space of functions into a special space of functions where some computations are simpler. In many cases, a backward transformation to the original space can be constructed which produces either the original function or its transformation. In fuzzy mathematics, typical examples of these transformation operators represent various powerset constructions (which are mostly generalizations of Zadeh's extension principle), or very general method called fuzzy transform introduced in [17], which covers a lot of others well-known constructions.

In this section we introduce the notion of transformation operators transforming structures $T(X)$ defined over an object $X$ of a category $\mathbf{K}$ to the structure $T(Y)$ defined over an object $Y$, where $\mathbf{T}=(T, \diamond, \eta)$ is a monad in a category $\mathbf{K}$ and where the transformation process is defined by a monadic T-relation from $X$ to $Y$.

We show that this generally defined transformation operator includes a number of known and often used transformations in fuzzy sets and fuzzy rough sets theory, including various generalizations of Zadeh's extension principle, transformations defined using lattice-valued fuzzy relations, and lattice-valued F-transform. Although the definitions of these operators are different and related to different fuzzy structures, we show that these transformation operators represent a method based on the existence of monadic relations in the respective categories. This universal approach to transformation operators makes it possible to unify the theory of these operators, allows by the selection of appropriate categories and monads in these categories to create new transformation operators, and using morphisms between monads it allows to find relationships between individual transformation operators.

In the following definition, we introduce a general notion of a transformation of an object $X$ of category $\mathbf{K}$ defined by a $\mathbf{T}$-relation in this category.

Definition 7. Let $\mathbf{T}=(T, \diamond, \xi)$ be a monad in a category $\mathbf{K}$ and let $R: X \rightsquigarrow Y$ be a $\mathbf{T}$-relation from $X$ to $Y$. Then an $R$-transformation of an object $X$ in an object $Y$ is a $\mathbf{K}$-morphism

$$
R^{\rightarrow}=R \diamond 1_{T(X)}: T(X) \rightarrow T(Y),
$$

where $1_{T(X)}$ is the identity morphism $T(X) \rightarrow T(X)$ in $\mathbf{K}$.
Using the same notation, we can simply calculate the composition of these monadic transformation operators. In fact, let $R: X \rightsquigarrow Y$ and $S: Y \rightsquigarrow Z$ be T-relations. Then for the composition $S \rightarrow \cdot R \rightarrow$ of transformation operators $R \rightarrow$ and $S \rightarrow$ we obtain

$$
\begin{gathered}
(S \diamond R)^{\rightarrow}=(S \diamond R) \diamond 1_{T(X)}=S \diamond\left(R \diamond 1_{T(X)}\right)= \\
S \diamond\left(1_{T(Y)} .\left(R \diamond 1_{T(X)}\right)\right)=\left(S \diamond 1_{T(Y)}\right) .\left(R \diamond 1_{T(X)}\right)=S^{\rightarrow} \cdot R^{\rightarrow} .
\end{gathered}
$$

Hence, the composition is also the transformation operator defined by a monadic relation $S \diamond R$.

Let us consider the following examples of transformation operators which are frequently used in fuzzy and rough set theory and also in fuzzy soft set theory.

The simplest example of this transformation operator is an upper transformation of a set defined by a relation which is well known from a generalized rough set theory based on a relation (see, e.g., [33]).

Example 6. Let $R \subseteq X \times Y$ be a relation. The transformation operator $R^{\Rightarrow}: 2^{X} \rightarrow 2^{Y}$ is defined by

$$
A \in 2^{X}, \quad R \Rightarrow(A)=\{y \in Y: \exists a \in A,(a, y) \in R\} .
$$

Another frequently used transformation operator is defined by a classical Zadeh's extension principle.

Example 7. Let $f: X \rightarrow Y$ be a mapping, then the $\mathcal{L}$-valued Zadeh's extension operator $f \Rightarrow: L^{X} \rightarrow L^{Y}$ is defined by

$$
f \Rightarrow(s)(y)= \begin{cases}\bigvee_{x \in X, f(x)=y} s(x), & \text { if } f^{-1}(y) \neq \varnothing, \\ 0, & \text { otherwise }\end{cases}
$$

where $s \in L^{X}, y \in Y$.

Example 8. In the fuzzy set theory the following transformation operator $R \Rightarrow: L^{X} \rightarrow L^{Y}$, based on a fuzzy relation $R: X \times Y \rightarrow L$ is standardly used in generalized fuzzy rough set theory:

$$
s \in L^{X}, y \in Y \quad R \Rightarrow(s)(y)=\bigvee_{x \in X} s(x) \otimes R(x, y)
$$

Some transformation operators $R \Rightarrow$ can also be defined such that $R \Rightarrow: C(X) \rightarrow C(Y)$ where $C(X) \subseteq L^{X}$. Let us consider the following simple example.

Example 9. Let $R$ be a relation from $(X, \delta)$ to $(Y, \gamma)$ from Example 3. The transformation operator $R^{\Rightarrow}: F(X, \delta) \rightarrow F(Y, \gamma)$ is formally defined in the same way as in (3). This transformation operator transforms fuzzy sets in $X$ extensional with respect to $\delta$, into fuzzy sets in $Y$, extensional with respect to $\gamma$.

Example 10. A well-known and frequently used transformation operator is the lattice-valued upper F-transform operator which in a lattice-valued form was introduced by Perfilieva [17]. This operator $F_{X, \mathcal{A}}^{\uparrow}: L^{X} \rightarrow L^{|\mathcal{A}|}$ is based on a space with a fuzzy partition $(X, \mathcal{A})$, where $\mathcal{A}=\left\{A_{\alpha}: \alpha \in|\mathcal{A}|\right\}$, i.e.,

$$
s \in L^{X}, \alpha \in|\mathcal{A}|, \quad F_{X, \mathcal{A}}^{\uparrow}(s)(\alpha)=\underset{x \in X}{\vee} s(x) \otimes A_{\alpha}(x) .
$$

This operator is used, among other things, as a significant cardinality reduction of the basic set $X$. Hence, it aggregates the original 
fuzzy sets defined in a large set $X$ to the fuzzy sets defined in the relatively small index set $|\mathcal{A}|$.

In the last example we show that operators of this type occur also in other structures than the standard fuzzy sets, namely in fuzzy soft sets.

Example 11. Let $U$ be a (fixed) set of objects and let $(E, R)$ be a fuzzy soft relation from $X$ to $Y$ defined in Example 5. Hence, $E \subseteq X \times Y$ is a relation from $X$ to $Y$ and $R: E \rightarrow L^{U}$ is an evaluation map. Using the notation from the Theorem 1 , by $T(X)$ we denote the set of all fuzzy soft sets $(A, s)$ from $X$, i.e., $A \subseteq X$ and $s: A \rightarrow L^{U}$. Then the transformation operator $(E, R) \Rightarrow: T(X) \rightarrow T(Y)$ is defined by

$$
\begin{gathered}
(E, S) \Rightarrow(A, s)=\left(E[A], s^{\Rightarrow}\right), \quad \text { where } \\
E[A]=\{y \in X: \exists x \in A,(x, y) \in E\}, s \Rightarrow: E[A] \rightarrow L^{U}, \\
y \in E[A], u \in U, \quad s \Rightarrow(y)(u)=\bigvee_{\{x \in A:(x, y) \in E\}} s(x)(u) \otimes S(x, y)(u) .
\end{gathered}
$$

This transformation operator can be used, e.g., in selective color segmentation problem (see, e.g., [26]).

Our main goal in this section is to show that although the transformation operators in each of the above presented examples are defined in different structures and using different formulas, all of these operators are, in fact examples of $R$-transformation operators defined for various monadic $R$-relations and categories and are defined by the universal formula.

Theorem 2. Let us consider an arbitrary example from Examples 6-11 and let $\mathcal{Q}: D(X) \rightarrow D(Y)$ be an transformation operator from this example. Then there exists a category $K$, a monad $\mathbf{D}=(D, \diamond, \eta)$ in $K$, and a D-relation $S$ such that

$$
\mathcal{Q}=S \rightarrow=S \diamond 1_{D(X)}
$$

Proof. Let us consider the following cases:

Case Example 6: Let $R \subseteq X \times Y$ be a relation from Example 6 . Let $\mathbf{K}=$ Set, $\mathbf{D}=\mathbf{P}=(P, \diamond, \eta)$ from Example 1 and let $S=\Phi(R): X \rightarrow P(Y)$, where $R$ is from Example 6. It can be verified by a simple calculation that $R \Rightarrow=S \diamond 1_{P(X)}$.

Case Example 7: Let $f: X \rightarrow Y$ be a map from Example 7. Let $\mathbf{K}=$ Set, $\mathbf{D}=\mathbf{Z}=(Z, \diamond, \chi)$ from Example 2 and let $S$ be defined by

$$
S=\ulcorner f\urcorner=\chi_{Y} \cdot f: X \rightarrow Z(Y),
$$

i.e., $S$ is a "graph" of a mapping $f$. For $s \in Z(X), y \in Y$ we have

$$
\begin{gathered}
S \rightarrow(s)(y)=\left(S \diamond 1_{Z(X)}\right)(s)(y)=\bigvee_{z \in X} 1_{Z(X)}(s)(z) \otimes \chi_{Y \cdot} f(z)(y)= \\
\bigvee_{z \in X} s(z) \otimes \chi_{Y,\{f(z)\}}(y)=\left\{\begin{array}{ll}
\bigvee_{z \in X, f(z)=y} s(z), & \text { iff } f^{-1}(y) \neq \varnothing \\
0_{L}, & \text { otherwise }
\end{array}=f \Rightarrow(s)(y) .\right.
\end{gathered}
$$

Therefore, $f \Rightarrow=\ulcorner f\urcorner \rightarrow=\ulcorner f\urcorner \diamond 1_{Z}(X)$.

Case Example 8: Let $\mathbf{K}=$ Set, $\mathbf{D}=\mathbf{Z}=(Z, \diamond, \chi)$ from Example 2 and let $S=\Phi(R)$, where $R$ is from Example 8. As can be easily seen, it holds $R \Rightarrow=S \rightarrow=S \diamond 1_{Z(X)}$.
Case Example 9: Let $R$ be a relation from $(X, \delta)$ to $(Y, \gamma)$ from Example 9 and let $\mathbf{K}=\operatorname{Set}(\mathcal{L}), \mathbf{D}=\mathbf{F}=(F, \diamond, \eta)$ be a monad from Example 3. According to Theorem 1, we set $S=\Phi(R)$ : $(X, \delta) \rightarrow\left(F(Y, \gamma), \sigma_{Y, \gamma}\right)$. As can be easily seen, it holds $R \Rightarrow=S \rightarrow=$ $S \diamond 1_{\mathcal{F}(X, \delta)}$.

Case Example 10: Let $(X, \mathcal{A})$ be a space with a fuzzy partition, $\mathcal{A}=$ $\left\{A_{i}: i \in|\mathcal{A}|\right\}$ and let $F_{X, \mathcal{A}}^{\uparrow}: L^{X} \rightarrow L^{|\mathcal{A}|}$ be the F-transform. We define a $\mathcal{L}$-valued relation $R: X \times|\mathcal{A}| \rightarrow \mathcal{L}$ by

$$
x \in X, i \in|\mathcal{A}|, \quad R(x, i)=A_{i}(x) .
$$

Then $R$ is a relation from Example 2 and according to Theorem 1 , $S=\Phi(R): X \rightarrow Z(|\mathcal{A}|)$ is a Z-relation in the category Set and for $s \in Z(X), i \in|\mathcal{A}|, S^{\rightarrow}(s)(i)=F_{X, \mathcal{A}}^{\uparrow}(s)(i)$.

Case Example 11: Let $(E, R)$ be a fuzzy soft relation from $X$ to $Y$ defined in Example 5 and let $S=\Phi(E, R)$ be a T-relation $X \rightarrow T(Y)$ from Theorem 1, i.e., we have

$$
\begin{gathered}
x \in X, S(x)=\Phi(E, R)(x)=\left(Y_{x}^{E}, R_{x}\right) \in T(Y), Y_{x}^{E}=\{y \in Y:(x, y) \in E\} \\
\forall y \in Y_{x}^{E}, R_{x}(y)(u)=R(x, y)(u) .
\end{gathered}
$$

We show that for arbitrary $(A, s) \in T(X)$ the following holds

$$
S^{\rightarrow}(A, s)=\left(\Phi(E, R) \diamond 1_{T(X)}\right)(A, s)=(E, R) \Rightarrow(A, s)=(E[A], s) .
$$

In fact, according to Theorem 1, Case Example 5, we obtain

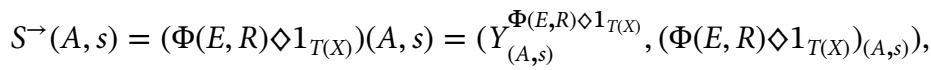
where

$$
\begin{gathered}
Y_{(A, s)}^{\Phi(E, R) \diamond 1_{T(X)}}=\bigcup_{\substack{1_{T(X)} \\
\operatorname{T}_{(A, s)}}} Y_{z}^{\Phi(E, R)}=\bigcup_{z \in A} Y_{z}^{\Phi(E, R)}=\bigcup_{z \in A} E[z]=E[A], \\
\left(\Phi(E, R) \diamond 1_{T(X)}\right)_{(A, s)}: E[A] \rightarrow L^{U}, b \in E[A], u \in U, \\
\left(\Phi(E, R) \diamond 1_{T(X)}\right)_{(A, s)}(b)(u)= \\
\bigvee_{z \in X_{(A, s)}^{1_{T(X)}}: b \in Y_{z}^{\Phi(E, R)}} 1_{T(X),(A, s)}(z)(u) \otimes \Phi(E, R)_{z}(b)(u)= \\
\bigvee_{z \in A: b \in E[z]} s(z)(u) \otimes R_{z}(b)(u)=s \Rightarrow(b)(u) .
\end{gathered}
$$

Therefore, $\Phi(E, R) \rightarrow=(E, R) \Rightarrow$.

Remark 1. Monadic relations have a wide range of applications even outside the traditional fuzzy structures. One way to apply this theory is the general theory of automata, which can be defined in general categories using monadic relations. In fact, let us recall the following definition from [31].

Definition 8. Let $\mathbf{K}$ be a category and let $\mathbf{T}=(T, \diamond, \eta)$ be a monad (in clone form) in $\mathbf{K}$. Then a $\mathbf{T}$-automaton in a category $\mathbf{K}$ is a system $(S,(M, *), \delta)$ such that

1. $S$ is an object of $\mathbf{K}$, which represents a set of automaton states.

2. $(M, *)$ is a monoid, representing automaton inputs.

3. $\delta:(M, *) \rightarrow\left(\operatorname{Rel}_{\mathbf{T}}(S), \diamond\right)$ is a monoid homomorphism, where $\mathrm{Rel}_{\mathbf{T}}$ is the set of $\mathbf{T}$-relations from $S$ to $S$, i.e., 
(a) For each $m \in M, \delta(m): S \rightarrow T(S)$ is a T-relation,

(b) $\delta\left(1_{M}\right)=\eta_{S}$,

(c) For each $m, n \in M, \delta(m * n)=\delta(n) \diamond \delta(m)$,

which represents an automaton transition function.

In Močkoř [31] it was proven that this definition comprises all wellknown types of automata, including deterministic, nondeterministic or $\mathcal{L}$-valued fuzzy automata. It is well known in automata theory that instead of the set (object) of states $S$, the powerset $P(S)$ of all subsets of $S$, the set of all fuzzy sets $F(S)$ in $S$, or another powerset structure $T(S)$ can be used depending on the type of an automaton. In this case, it is necessary to extend the function $\delta$ such that instead of a morphism $\delta(m): S \rightarrow T(S)$, the morphism $\bar{\delta}(m): T(S) \rightarrow T(S)$ is used. This extended morphism was mostly defined individually for each type of automaton and required verification of a number of properties. Using the theory of $\mathbf{T}$-transformations, however, we can define this extension $\bar{\delta}$ using one formula as follows:

$$
m \in M, \quad \bar{\delta}(m)=\delta(m) \rightarrow=\delta(m) \diamond 1_{T(S)}
$$

Such a universally defined extension $\bar{\delta}$ of the transition function $\delta$ includes extensions for all standard types of automata, without the need to individually define these extensions for individual types of automata.

\section{CONCLUSIONS}

The main goal in this paper was to show that many of the standardly used transformation operators in categories of fuzzy structures are in fact universal transformation operators defined by one formula using different monadic relations in these categories. For this purpose we used the idea of L.A.Manes and introduced the concept of a relation $R$ in the category $\mathbf{K}$ defined by a monad $\mathbf{T}=(T, \diamond, \eta)$ as a morphism $R: X \rightarrow T(Y)$. Using properties of a monad $\mathbf{T}$, this morphism can be extended to a morphism $R^{\rightarrow}: T(X) \rightarrow T(Y)$, where $R^{\rightarrow}=R \diamond 1_{T(X)}$ and this extended morphism can be considered a transformation operator from an object $X$ to the object $Y$ defined by a $\mathbf{T}$-relation $R$. It seems that this general approach to the issue of relations and transform operators defined by them has many concrete examples in various categories of fuzzy objects. This approach can be also used in others areas, including, e.g., the theory of automata of various types where it allows to unify some procedures performed up to now individually for different types of automata [31].

For further research in this field, it will be useful to focus on examining other general properties of these transformation operators defined by monadic relations, and on the research between these operators on one hand and the respective monads and monadic relations on the other.

The development potential of relations defined by monads and their transformation operators lies, among other things, in methods of constructing monads in categories. For each $\operatorname{monad} \mathcal{T}=(T, \diamond, \eta)$ in a category $\mathbf{K}$ there exist a category $\mathbf{L}$ and functors $F: \mathbf{K} \rightarrow \mathbf{L}$ and $G: \mathbf{L} \rightarrow \mathbf{K}$ such that $T=G$.F and the functors $F$ and $G$ are adjoint. For possible applications of the theory of monadic relations, however, the reverse implication of this statement is of key importance, i.e., for any pair $F$ and $G$ of adjoint functors between two categories, the functor $T=G . F$ is a functor from the monad. Since the saying "adjunctions occur everywhere" is well known in the category theory and mathematics, using suitable categories $\mathbf{K}$ representing "fuzzy objects" (such as categories $\operatorname{Set}(\mathcal{L})$ or SpaceFP, e.g.), different pairs of adjoint functors can be found between these categories and some other categories (or subcategories) that form an adjunction. In this way we can obtain completely new monads, monadic relations, and the corresponding transformations in this category K. Thus, this theory of monads and monadic relations can be used as a very useful source of new examples of transformation operators over fuzzy objects.

\section{CONFLICTS OF INTEREST}

Author declare that he has no potential conflict of competing interest.

\section{ACKNOWLEDGMENTS}

This research was partially supported by the project $18-06915 \mathrm{~S}$ provided by the Grant Agency of the Czech Republic.

\section{REFERENCES}

[1] L.A. Zadeh, Fuzzy sets, Inf. Control. 8 (1965), 338-353.

[2] M. Barr, Fuzzy sets and topos theory, Canad. Math. Bull. 29 (1986), 501-508.

[3] U. Höhle, L.N. Stout, Foundations of fuzzy sets, Fuzzy Sets Syst. 40 (1991), 257-296.

[4] L.N. Stout, Topoi and categories of fuzzy sets, Fuzzy Sets Syst. 12 (1984), 169-184.

[5] L.N. Stout, A survey of fuzzy sets and topos theory, Fuzzy Sets Syst. 42 (1991), 3-14.

[6] J. Harding, C. Walker, E. Walker, Categories with fuzzy sets and relations, Fuzzy Sets Syst. 256 (2014), 149-165.

[7] U. Höhle, M-Valued sets and sheaves over integral commutative cl-monoids, in: S.E. Rodabaugh et al. (Eds.), Applications of Category Theory to Fuzzy Subsets, Springer, Dordrecht, Netherlands, 1992, pp. 33-72.

[8] U. Höhle, GL-quantales: Q-valued sets and their singletons, Stud. Logica. 61 (1998), 123-148.

[9] U. Höhle, T. Kubiak, Quantale Sets and Their Singleton Monad, Preprint, 2008.

[10] S.E. Rodabaugh, Powerset operator foundation for poslat fuzzy set theories and topologies, in: U. Höhle, S.E. Rodabaugh (Eds.), Mathematics of Fuzzy Sets: Logic, Topology and Measure Theory, The Hnadbook of Fuzzy Sets Series, vol. 3, Springer, Boston, MA, USA, 1999, pp. 91-116.

[11] S.E. Rodabaugh, Powerset operator based foundation for point-set lattice theoretic (poslat) fuzzy set theories and topologies, Quaest. Math. 20 (1997), 463-530.

[12] S.A. Solovyov, On the category set (JCPos), Fuzzy Sets Syst. 157 (2006), 459-465.

[13] S.A. Solovyov, Categories of lattice-valued sets as categories of arrows, Fuzzy Sets Syst. 157 (2006), 843-854.

[14] S.A. Solovyov, Powerset operator foundations for catalg fuzzy set theories, Iran. J. Fuzzy Syst. 8 (2001), 1-46. 
[15] J.A. Goguen, L-fuzzy sets, J. Math. Anal. Appl. 18 (1967), 145-174.

[16] I. Perfilieva, A.P. Singh, S.P. Tiwari, On the relationships among F-transform, fuzzy rough sets and fuzzy topology, Soft Comput. 21 (2017), 3513-3523.

[17] I. Perfilieva, Fuzzy transforms: theory and applications, Fuzzy Sets Syst. 157 (2006), 993-1023.

[18] C.Y. Wang, Fuzzy rough sets based on generalized residuated lattices, Inf. Sci. 248 (2013), 31-49.

[19] W.Z. Wu, Y. Leung, J.S. Mi, On characterization of (J, T)-fuzzy rough approximation operators, Fuzzy Sets Syst. 154 (2005), 76-102.

[20] L.A. Manes, Book review fuzzy sets and systems, theory and applications, Bull. Am. Math. Soc. 7 (1982), 603-612.

[21] V. Novák, I. Perfilijeva, J. Močkoř, Mathematical Principles of Fuzzy Logic, Springer, Boston, MA, USA, 1999.

[22] J. Močkoř, Spaces with fuzzy partitions and fuzzy transform, Soft Comput. 21 (2017), 3479-3492.

[23] I. Perfilieva, A.P. Singh, S.P. Tiwari, On the relationship among Ftransform, fuzzy rough set and fuzzy topology, in Proceedings of IFSA-EUSFLAT, Atlantis Press, Amsterdam, Netherlands, 2015, pp. 1324-1330.

[24] J. Močkoř, M. Holčapek, Fuzzy objects in spaces with fuzzy partitions, Soft Comput. 21 (2017), 7269-7284.
[25] K. Maji, R. Biswas, A. Roy, Fuzzy soft sets, J. Fuzzy Math. 9 (2001), 589-602.

[26] J. Močkoř, P. Hurtik, Approximations of Fuzzy Soft Sets by Fuzzy Soft Relations with Image Processing Application, submitted to Soft Computing.

[27] E.G. Manes, Algebraic Theories, Springer Verlag, Berlin, Germany, 1976.

[28] H. Kleisli, Every standard construction is induced by a pair of adjoint functors, Proc. Am. Math. Soc. 16 (1965), 544-546.

[29] S.E. Rodabaugh, Relationship of algebraic theories to powerset theories and fuzzy topological theories for lattice-valued mathematics, Int. J. Math. Math. Sci. 2007 (2007), 1-71.

[30] J. Močkoř, Powerset operators of extensional fuzzy sets, Iran. J. Fuzzy Syst. 15 (2018), 143-163.

[31] J. Močkoř, Monads and a common framework for fuzzy type automata, Int. J. Gen. Syst. 48 (2019), 406-442.

[32] J. Močkoř, Relational variants of categories of fuzzy sets defined by monads, in The 11th Conference of the European Society for Fuzzy Logic and Technology: Proceedings of the 11th Conference of the European Society for Fuzzy Logic and Technology (EUSFLAT 2019), Atlantis Press, Praha, Czech Republic, 2019.

[33] Z. William, Generalized rough sets based on relations, Inf. Sci. 177 (2007), 4997-5011. 